\section{Schützt Kaffee vor Endometrium-Karzinom?}

\begin{abstract}
Aus epidemiologischen Studien sind für das Endometrium-Karzinom eine Reihe von Risikofaktoren bekannt. In einer Metaananalyse von mehreren Studien wurde nun der Frage nachgegangen, wie sich ein erhöhter Kaffeegenuss auf das Risiko für ein Endometrium-Karzinom auswirkt.
\end{abstract}

$\mathrm{D}$ as Endometrium-Karzinom ist eine der häufigsten gynäkologischen Neoplasien. Epidemiologische Studien konnten einen Zusammenhang des Endometrium-Karzinoms mit Adipositas sowie erhöhten Östrogen- und Insulinkonzentrationen belegen, die das Wachstum von endometrialen Zellen fördern. Regelmäßige körperliche Aktivität gilt als wichtige Vorbeugung vor einem Endometrium-Karzinom. Auch lässt sich das Risiko durch bestimmte Diätmaßnahmen senken. Kaffee, sicher eines der weltweit am meisten genossenen $\mathrm{Ge}$ tränke, setzt sich aus vielen Substanzen zusammen, von denen einige antimutagene und antioxidative Eigenschaften besitzen. Studien wiesen darauf hin, dass Kaffee hormonmodulierende Effekte hat. Es wurde gezeigt, dass Kaffeegenuss die Spiegel von Östrogen sowie CPeptid senkt. Mehrere epidemiologische Studien wurden in den letzten Jahren mit der Frage einer möglichen Assoziation von Kaffeegenuss und dem Risiko für ein Endometrium-Karzinom durchgeführt. In einer Metaanalyse wurden jetzt 16 Beobachtungsstudien aus den USA, Europa und Japan ausgewertet, die bis Oktober 2011 publiziert wurden. Von den 16 Studien waren zehn Fall-Kontrollund 6 Kohortenstudien. Insgesamt umfassten die Studien 6.628 Fälle. Das gepoolte relative Risiko für ein Endometrium-Karzinom für die höchste (drei bis vier Tassen/Tag) versus der niedrigsten aufgenommenen Kaffeemenge betrug 0,71 (95\% Konfidenzintervall (KI): 0,61$0,81 ; \mathrm{p}=0,13)$. Bezogen auf das Studiendesign betrug das gepoolte relative Risiko 0,69 (95\% KI: 0,55-0,87) für FallKontroll-Studien und 0,70 (95\% KI: 0,61-0,80) für Kohortenstudien. Bei Studien aus Japan war diese inverse Assoziation stärker ausgeprägt (relatives Risiko (RR): 0,40 ; $95 \%$ KI: $0,25-0,63$ ) als bei den US-Studien (RR: 0,69; 95\% KI: 0,600,79 ) und Europa (RR: 0,79; 95\% KI: $0,63-0,99$ ). Eine zusätzliche Tasse Kaffee pro Tag bewirkte ein gepooltes relatives Risiko von 0,92 (95\% KI: 0,90-0,95), d. h. eine zusätzliche Tasse Kaffee senkte das Risiko für ein Endometrium-Karzinom um 8\%. Unabhängig vom Studiendesign zeigte sich, dass ein erhöhter Kaffeegenuss mit einem reduzierten Risiko für ein Endometrium-Karzinom einherging.

Fazit: Wie diese Metaanalyse zeigt, nimmt das Risiko für ein EndometriumKarzinom mit zunehmendem Kaffeegenuss ab. Auch bestätigt diese Studie frühere Annahmen eines präventiven Effektes von Kaffee für die Entstehung eines Endometrium-Karzinoms. Bei drei bis vier Tassen Kaffee/Tag nimmt das Risiko für ein Endometrium-Karzinom um $29 \%$ ab. Einen Lebensmittelkonzern mit Sitz in der Schweiz werden diese Ergebnisse sicher freuen. Die Autoren von der Harvard School of Public Health gaben keinen Interessenkonflikt an.

Prof. Dr. Tino F. Schwarz

JE Y et al.: Coffee consumption and risk of endometrial cancer: findings from a large up-to-date meta-analysis. Int J Cancer 2012; 131: 1700-10

\title{
Dichte Brust macht Brustkrebs nicht tödlicher
}

Teilentwarnung für Frauen mit mammografisch dichter Brust: Sie haben zwar ein stark erhöhtes Risiko, an Brustkrebs zu erkranken, aber sie sterben nicht häufiger daran als Frauen mit weniger dichtem Drüsengewebe.

$U_{\mathrm{H}}^{\mathrm{n}}$ $\mathrm{m}$ den Faktor vier bis fünf ist bei Frauen mit dichtem Brustdrüsengewebe die Brustkrebsrate erhöht. Die Tumoren haben zudem häufig prognostisch ungünstige Eigenschaften. Damit könnte Brustkrebs bei Frauen mit dichter Brust mit einer höheren Mortalität assoziiert sein. Dieser Vermutung wurde nun in einer Studie nachgegangen. Die Autoren werteten die Daten von 9.232 Frauen aus, die zwischen 1996 und 2005 mit der Diagnose eines primären invasiven Mammakarzinoms im US Breast Cancer Surveillance Consortium registriert worden waren. $84 \%$ hatten nach den Kriterien des American College of Radiology (ACR) Brüste mit mittleren Dichtegra-

den (ACR 2 oder 3), 10\% extrem dichtes Drüsengewebe (ACR 4).

Bei ACR-4-Frauen war der Krebs häufiger im Intervall aufgetreten, hatte öfter Lymphknoten befallen und wurde eher mit einer Chemotherapie behandelt. ACR1-Frauen hatten dagegen ein etwas höheres Risiko, dass der Tumor bei Diagnose schon im AJCC-Stadium IV vorlag. Während der mittleren Beobachtungszeit von 6,6 Jahren starben 1.795 Frauen, davon 889 infolge des Krebses. Wurden Lokalisation und Stadium des Karzinoms, Alter und Jahr bei Diagnose, BMI, Art der Entdeckung und Therapie berücksichtigt, hatten ACR-4- im Vergleich zu ACR2-Frauen weder eine höhere Brustkrebs- sterblichkeit $(\mathrm{HR}=0,92,95 \%-\mathrm{KI}=0,71-$ $1,19)$ noch eine höhere Sterblichkeit insgesamt $(\mathrm{HR}=0,83,95 \%-\mathrm{KI}=0,68-1,02)$. Eine gegenüber Frauen mit ACR 2 erhöhte Brustkrebssterblichkeit zeigte sich jedoch bei ACR-1-Frauen - sofern sie adipös waren $(\mathrm{HR}=2,02)$, Tumoren von mind. 2 $\mathrm{cm}(\mathrm{HR}=1,55)$ oder im fortgeschrittenen Stadium hatten $(\mathrm{HR}=1,45)$. Erklärung laut Autoren: Der hohe Fettanteil der Brust könnte eine für Tumorwachstum und -progression günstige Mikroumgebung sein. Für Brustkrebspatientinnen mit extrem dichter Brust sei das Ergebnis dagegen „beruhigend“. Offenbar sind Risikofaktoren für Brustkrebs nicht notwendig auch Faktoren, die die Prognose beeinträchtigen, wenn sich bereits ein Karzinom entwickelt hat.

Dr. Beate Schumacher

Gierach G. et al. Relationship Between Mammographic Density and Breast Cancer Death in the Breast Cancer Surveillance Consortium. JNCI 2012; online 20. August, DOI:10.1093/jnci/djs327 